See discussions, stats, and author profiles for this publication at: https://www.researchgate.net/publication/343147322

\title{
Optimizing the impact of medications for opioid use disorder at release from prison and jail settings: A microsimulation modeling study
}

Article in International Journal of Drug Policy · July 2020

DOI: 10.1016/j.drugpo.2020.102841

\section{CITATIONS}

9 authors, including:

Alexandria Macmadu

Brown University

24 PUBLICATIONS 182 CITATIONS

SEE PROFILE

s. Bessey

Brown University

5 PUBLICATIONS 1 CITATION

SEE PROFILE
READS

90

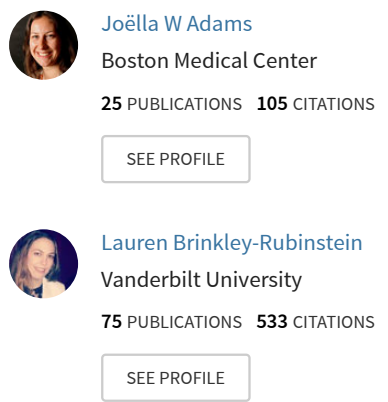

Some of the authors of this publication are also working on these related projects:

Rhode Island Prescription and Illicit Drug Study (RAPIDS): Responding to Fentanyl and Associated Harms View project

Drug Overdose Surveillance and Information System: PreventOverdoseRI.org (PORI) View project 


\title{
Optimizing the impact of medications for opioid use disorder at release from prison and jail settings: A microsimulation modeling study
}

\author{
Alexandria Macmadu $^{\mathrm{a}, \mathrm{b}}$, Joëlla W. Adams ${ }^{\mathrm{a}}$, S.E. Bessey ${ }^{\mathrm{a}}$, Lauren Brinkley-Rubinstein ${ }^{\mathrm{c}, \mathrm{d}}$, \\ Rosemarie A. Martin ${ }^{\mathrm{e}}$, Jennifer G. Clarke ${ }^{\mathrm{f}}$, Traci C. Green ${ }^{\mathrm{a}, \mathrm{g}}$, Josiah D. Rich ${ }^{\mathrm{a}, \mathrm{b}}$, \\ Brandon D.L. Marshall ${ }^{\mathrm{a}, *}$ \\ a Department of Epidemiology, Brown University School of Public Health, 121 South Main Street, Providence, RI 02903, USA \\ ${ }^{\mathrm{b}}$ The Center for Prisoner Health and Human Rights, The Miriam Hospital, 8 Third Street, Providence, RI 02906, USA \\ ${ }^{\mathrm{c}}$ Department of Social Medicine, University of North Carolina at Chapel Hill, 333 South Columbia Street, Chapel Hill, NC 27516, USA \\ ${ }^{\mathrm{d}}$ Center for Health Equity Research, University of North Carolina at Chapel Hill, 335 South Columbia Street, Chapel Hill, NC 27514, USA \\ ${ }^{\mathrm{e}}$ Department of Behavioral and Social Science, Brown University School of Public Health, 121 South Main Street, Providence, RI 02903, USA \\ ${ }^{\mathrm{f}}$ Rhode Island Department of Corrections, 40 Howard Avenue, Cranston, RI 02920, USA \\ ${ }^{\mathrm{g}}$ Department of Emergency Medicine, The Warren Alpert School of Medicine of Brown University, Rhode Island Hospital, 55 Claverick Street, Providence, RI 02903, USA
}

A R T I C L E I N F O

\section{Keywords:}

Opioids

Overdose

Medications for opioid use disorder

Prisons

Jails

\begin{abstract}
A B S T R A C T
Background: We examined the impact of expanded access to medications for opioid use disorder (MOUD) in a unified prison and jail system on post-release, opioid-related overdose mortality.

Methods: We developed a microsimulation model to simulate a population of 55,000 persons at risk of opioidrelated overdose mortality in Rhode Island. The effect of an extended-release (XR) naltrexone only intervention and the effect of providing access to all three MOUD (i.e., methadone, buprenorphine, and XR-naltrexone) at release from incarceration on cumulative overdose death over eight years (2017-2024) were compared to the standard of care (i.e., limited access to MOUD).

Results: In the standard of care scenario, the model predicted 2385 opioid-related overdose deaths between 2017 and 2024. An XR-naltrexone intervention averted 103 deaths (4.3\% reduction), and access to all three MOUD averted 139 deaths (5.8\% reduction). Among those with prior year incarceration, an XR-naltrexone only intervention and access to all three MOUD reduced overdose deaths by $22.8 \%$ and $31.6 \%$, respectively.

Conclusions: Expanded access to MOUD in prison and jail settings can reduce overdose mortality in a general, atrisk population. However, the real-world impact of this approach will vary by levels of incarceration, treatment enrollment, and post-release retention.
\end{abstract}

\section{Introduction}

The epidemic of opioid-related overdose mortality is a public health crisis that continues to evolve across the US. In 2018, there were over 67,000 overdose deaths documented in the US, and approximately $70 \%$ were opioid-involved (Wilson, Kariisa, Seth, Smith \& Davis, 2020). From 2015 to 2016 alone, drug overdose deaths increased by $21 \%$ nationwide, and the emergence of fentanyl in drug markets has contributed to dramatic increases in overdose mortality in several states (Scholl, Seth, Kariisa, Wilson \& Baldwin, 2019).

The risk of opioid-related overdose mortality is particularly acute among persons with recent incarceration in prisons and jails
(Binswanger et al., 2007; Binswanger, Blatchford, Mueller \& Stern, 2013; Merrall et al., 2010). During incarceration, tolerance is diminished or lost, which places individuals at greatly elevated risk of overdose upon release (Møller et al., 2010). Disrupted social support networks, limited or interrupted access to medications for opioid use disorder (MOUD) in the community, medical comorbidities, and a variety of other socioeconomic conditions further exacerbate overdose risk among individuals leaving incarceration (Joudrey et al., 2019). In a seminal paper, Binswanger et al. found that the first two weeks following release from incarceration were associated with a 129 -fold increase in overdose risk relative to the general population (Binswanger et al., 2007).

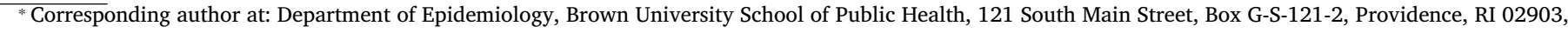
USA.

E-mail address: brandon_marshall@brown.edu (B.D.L. Marshall).
} 
Increased access to MOUD for persons who are incarcerated is a promising approach to reduce mortality and overdose risk following release from incarceration (Hedrich et al., 2012; National Academies of Sciences \& Medicine, 2019). A recent meta-analysis and systematic review of the effectiveness of MOUD in prison and jail settings found that the provision of MOUD in prisons and jails reduced non-medical opioid use and increased treatment entry in the community post-release (Moore et al., 2019). While additional medical, social, and economic benefits to expanding access to MOUD to persons who are incarcerated have been documented (Heimer et al., 2006; Kinlock, Gordon, Schwartz, Fitzgerald \& O'Grady, 2009; Lee et al., 2016, 2018; Rich et al., 2015; Zaller et al., 2013), access to MOUD for persons who are incarcerated has been very limited in the US.

In 2016, the Rhode Island Department of Corrections (RIDOC) became the first statewide correctional system in the US to provide comprehensive access to MOUD to persons who are incarcerated (Clarke, Martin, Gresko \& Rich, 2018). The program provides access to all three US Food and Drug Administration-approved MOUD (i.e., methadone, buprenorphine, XR-naltrexone), screens all persons who become incarcerated for opioid use disorder, initiates and continues MOUD for medically eligible individuals who choose to do so, and connects individuals to MOUD providers in the community at release (Clarke et al., 2018). Prior to 2016, the standard of care at RIDOC was to provide access to methadone only to pregnant women and to individuals who were enrolled in treatment with methadone immediately prior to incarceration-the latter of which was limited to an approximately 30-day taper protocol (Clarke et al., 2018). The pre-2016 standard of care in Rhode Island represents an important base of comparison from a policy-making perspective, as limited MOUD access in prison and jail settings is a national norm (Vestal, 2018).

Providing access to all three MOUD to incarcerated persons is a demonstrated overdose prevention approach, but the long-term, broader impact on overdose mortality in the larger at-risk population is unknown. In this study, microsimulation modeling was used to quantify the potential effect of expanded MOUD access in a combined prison and jail setting on overdose mortality in a population of at-risk persons in Rhode Island, USA, a state with a high burden of overdose mortality (i.e., an estimated 25.9 opioid overdose deaths per 100,000 in 2018) (Wilson et al., 2020). Microsimulation modeling is an ideal methodological approach for the current research question because it permits evaluation of multiple counterfactual scenarios that could not be implemented concurrently and compared in the real world (Arnold, Harrison, Heppenstall \& Gilthorpe, 2019). This type of modeling is useful from a policymaking standpoint because it provides evidence for the long-term, population-level impact of public health interventions. The primary objective of this study was to measure the potential effect of providing MOUD access in a combined prison and jail setting on overdose mortality among all persons at risk of overdose in Rhode Island over eight years, as compared to an XR-naltrexone only intervention and the pre-2016 standard of care in the correctional setting.

\section{Methods}

\section{Model setting and structure}

Opioid-related overdose mortality over an 8-year period (2017-2024) was modeled using microsimulation. The model run period was constrained to 8 years to minimize future uncertainty while providing long-term projections that may be useful for policymaking. Agents in the model $(n=55,000)$ represent a population of individuals aged 13 and older in Rhode Island who are at risk of opioid overdose mortality. We defined persons at risk of opioid-related overdose mortality as all those residing in Rhode Island who used any opioid extramedically in the prior year and persons enrolled in treatment with MOUD in the prior year. Additional details regarding construction and estimation of the simulated population are provided in the Supplemental Appendix.

At model initialization, agents were ascribed time-varying and timefixed characteristics. Gender and injection drug use (IDU) status were fixed characteristics assigned to each agent in the model, based on estimates from the Substance Abuse and Mental Health Data Archive, statewide data, and existing literature (appendix pp. 5-7). Overdose mortality risk is expected to vary across the at-risk population, with some agents being characterized as being lower risk due to underlying behaviors (e.g., infrequent use, use in small amounts) and some agents being characterized as higher risk (e.g., homelessness, heavy use, IDU). We attempt to capture this heterogeneity in underlying overdose risk by assigning a base risk of opioid-related overdose mortality $\left(p_{x}\right)$ to four agent classes (male IDU, male non-IDU, female IDU, and female nonIDU), which are then influenced by dynamic agent states. In the current model, IDU status was used as a proxy for increased risk for overdose mortality over the lifetime of the model (Havens et al., 2011; Kinner et al., 2012; Mathers et al., 2013).

Dynamic states were determined by probabilities at each discrete time-step and were informed by statewide data and existing literature (appendix pp. 8). These dynamic agent states were: (1) underlying baseline overdose risk in the absence of MOUD or incarceration assigned to each agent class, (2) incarceration status (i.e., currently incarcerated, post-release, or no prior-year incarceration), and (3) MOUD status (methadone/buprenorphine, XR-naltrexone, or none). The model proceeds in a series of two-week discrete time-steps. The microsimulation model simulated a discrete-time stochastic model in steady state, in which agents can exit the model due to death or no longer being at risk of overdose mortality; the latter exit mechanism was implemented to represent the spontaneous cessation of extra-medical opioid use.

Agents did not return to the population after exiting the model. To maintain a steady state, agents exiting the model were replaced by agents belonging to the same time-invariant agent class (i.e., gender and IDU status). The model was calibrated to reproduce trajectories observed in overdose mortality, incarceration, MOUD treatment engagement (both at release from incarceration and overall in the community) in Rhode Island between 2014 and 2018. Further details regarding model calibration (pp. 18), processes, and parameterization are available in Table 1 and the Supplemental Appendix.

Pathways for agent transitions across dynamic states in the model in the standard of care (base case) are summarized in Fig. 1. Pathways in the model are identical for all agent classes; thus, gender and IDU status are not specified in Fig. 1. In the XR-naltrexone-only scenario, agents may enter treatment with XR-naltrexone directly from incarceration; therefore, in a schematic representing the XR-naltrexone-only scenario, an additional arrow from incarceration to XR-naltrexone would exist. In the all three MOUD scenario, agents may enter treatment with XRnaltrexone or methadone/buprenorphine directly from incarceration; therefore, in a schematic representing the all three MOUD scenario, additional arrows from incarceration to XR-naltrexone and from incarceration to methadone/buprenorphine would exist (see appendix pp. 8).

\section{Opioid-related overdose mortality}

Baseline risk for overdose mortality was determined by membership in both specific time-invariant and dynamic agent classes, with the relative risk of overdose mortality $\left(p_{\mathrm{x}}\right)$ assigned for IDU males $\left(p_{1}\right)$, nonIDU males $\left(p_{2}\right)$, IDU females $\left(p_{3}\right)$, and non-IDU females $\left(p_{4}\right.$; see appendix pp. 9). Baseline risk across agent classes was informed by statewide overdose decedent data (Prevent Overdose RI, 2019c). Agents that were neither engaged in MOUD nor incarcerated at a given time step were assigned a baseline relative risk of overdose mortality of $p_{\mathrm{x}}$. We used evidence from two systematic reviews (Degenhardt et al., 2011; Mathers et al., 2013) and additional literature (Krupitsky et al., 
Table 1.

Key model processes and parameters for a microsimulation model simulating overdose risk in a virtual population representing people at risk of overdose in Rhode Island, USA.

\begin{tabular}{ll}
\hline Parameter & Description \\
\hline $\begin{array}{l}\text { Demography } \\
\text { Population size }\end{array}$ & $n=55,000$ \\
Sex \& IDU status & $\begin{array}{l}\text { Distribution of male non-IDU (49.1\%), male IDU (14.5\%), } \\
\text { female non-IDU (32.7\%), and female IDU (3.6\%) at risk of } \\
\text { opioid-related mortality (age } 13+)\end{array}$
\end{tabular}
opioid-related mortality (age $13+$ )

Opioid-related overdose mortality Base risk

Release from incarceration

XR-naltrexone discontinuation
Methadone/buprenorphine
discontinuation
Methadone/buprenorphine
enrollment
XR-naltrexone enrollment

Incarceration

Incarceration

Sentence duration

Recidivism risk (3-year)

MOUD

MOUD initiation in the community (by agent class)

MOUD initiation in the community (by medication type)

MOUD initiation at RIDOC (by medication type)

Methadone/ buprenorphine distribution of treatment duration

XR-naltrexone distribution of treatment duration
The base risk of opioid related overdose mortality $\left(p_{\mathrm{x}}\right)$ was stratified by agent class (i.e., gender and IDU status). Overdose mortality then varies according to dynamic agent states, including MOUD enrollment vs. discontinuation status and incarceration recency.

$108 p$ at 2 weeks post-incarceration and then degrades with time (see appendix pp. 9)

$8 p$ at 2 weeks post-discontinuation

$2 p$ at 2 weeks post-discontinuation

$0.4 p$ while engaged in treatment

$0.2 p$ while engaged in treatment

Proportion of at risk population incarcerated per 2-week timestep: $955 / 55,000=1.7 \%$

Female distribution: 40\% 2-4 weeks, 47.5\% 5-26 weeks, 6.5\% 27-52 weeks, $4.5 \%$ 53-156 weeks ( 6 years), $1.5 \%>6$ years Male distribution: $43 \% 2-4$ weeks, 50\% 5-26 weeks, 2\% 27-52, $2 \%$ 53-156 (6 years), $3 \%>6$ years.

Varies by male $(51 \%)$ vs. female $(36 \%)$ agent class

Probability of initiating MOUD in the community at each timestep (applies to all scenarios): female non-IDU (1.25\%), male non-IDU (1.1\%), female IDU (8.1\%), male IDU (14.9\%)

Among those initiating MOUD, probability of initiating: XRnaltrexone (0.8\%), methadone/buprenorphine (99.2\%) Applies to all scenarios

Standard of care scenario: XR-naltrexone $(0 \%)$, methadone/ buprenorphine $(0 \%)$. XR-naltrexone only scenario: XRnaltrexone (66\%), methadone/buprenorphine $(0 \%)$. All three MOUD scenario: XR-naltrexone $(0.8 \%)$, methadone/ buprenorphine (99.2\%)

Distribution: 2 weeks $(6.7 \%), 4$ weeks $(6.2 \%), 6$ weeks (4\%), 8 weeks (4\%), 12 weeks (8.2\%), 18 weeks (6.5\%), 26 weeks (10.6\%), 52 weeks (18.8\%), $\geq 2$ years (35\%). Median duration: 52 weeks

Distribution: 4 weeks (45\%), 8 weeks (10\%), 12 weeks (9\%), 18 weeks (8\%), 26 weeks (8\%), $\geq 1$ year (20\%). Median duration: 8 weeks
Sources

Calculated using data from the Substance Abuse and Mental Health Data Archive: 2-Year Restricted-use Data Analysis System (2015-2016) (Substance Abuse \& Mental Health Data Archive, 2015-2016)

Calculated using data from the Substance Abuse and Mental Health Data Archive: 2-Year Restricted-use Data Analysis System (2015-2016) (Substance Abuse \& Mental Health Data Archive, 2015-2016); relevan literature (Fingerhood, King, Brooner \& Rastegar, 2014); and publicly available aggregate statewide MOUD utilization data (Prevent Overdose RI, 2019b)

Informed by publicly available aggregate statewide overdose data from the Rhode Island Department of Health (Rhode Island Department of Health, 2019), calibrated

Informed by publicly available aggregate statewide overdose data from the Rhode Island Department of Health (Rhode Island Department of Health, 2019) and relevant literature (Binswanger et al., 2013, 2007; Merrall et al., 2010), calibrated

Informed by relevant literature (Digiusto et al., 2004; Gibson et al., 2007; Saucier, Wolfe \& Dasgupta, 2018), calibrated

Informed by relevant literature (Sordo et al., 2017), calibrated

Informed by relevant literature (Degenhardt et al., 2011; Mathers et al., 2013), calibrated

Informed by relevant literature (Krupitsky et al., 2011; Larochelle et al., 2018), calibrated

Informed by Rhode Island Department of Corrections reports (Rhode Island Department of Corrections, 2017) and relevant literature (Lam et al., 2019), calibrated

Informed by Rhode Island Department of Corrections reports (Rhode Island Department of Corrections, 2017), calibrated

Informed by Rhode Island Department of Corrections reports (Rhode Island Department of Corrections, 2017)

Informed by publicly available statewide MOUD utilization data (Prevent Overdose RI, 2019b) and estimates from Substance Abuse and Mental Health Services Administration (SAMHSA) (Substance Abuse \& Mental Health Data Archive, 2015-2016) to achieve steady state Informed by publicly available aggregate statewide MOUD utilization data (Prevent Overdose RI, 2019b)

Informed by publicly available aggregate statewide MOUD utilization data (Prevent Overdose RI, 2019b) and relevant literature (Di Paola et al., 2014)

Informed by SAMHSA Treatment Episode Data Set - Discharges (TEDS-D), 2016 Rhode Island and relevant literature (Lee et al., 2016, 2018)

Informed by SAMHSA Treatment Episode Data Set - Discharges (TEDS-D), 2016 Rhode Island and relevant literature (Lee et al., 2016, 2018)
2011; Larochelle et al., 2018) to define the relative risk of overdose mortality during periods of MOUD enrollment, which was set to $0.4 p_{\mathrm{x}}$ and $0.2 p_{\mathrm{x}}$ for those enrolled in treatment with methadone/buprenorphine and XR-naltrexone, respectively. Based on prior literature, the relative risk of overdose mortality increased following MOUD discontinuation was set to $2 p_{\mathrm{x}}$ and $8 p_{\mathrm{x}}$ at 2 weeks post-discontinuation for those enrolled in methadone/buprenorphine and XR-naltrexone, respectively; the relative risk of overdose mortality then returned to $1 p_{\mathrm{x}}$ for a given agent thereafter. The relative risk of overdose mortality also increased following release from incarceration to $108 p_{\mathrm{x}}$ at 2 weeks post incarceration, then declined with time to $1 p_{\mathrm{x}}$ over the course of one year (see appendix pp. 9). The influence of dynamic agent states on baseline risk of overdose mortality across agent classes is summarized in Fig. 2.

\section{Incarceration}

The model proceeds in 2-week time-steps; therefore, incarceration was defined as being incarcerated in Rhode Island's unified prison and jail system for a period of at least 2 weeks. At each time-step, the mean distribution of agents who were incarcerated was as follows: $60.6 \%$ of non-IDU males, $27.8 \%$ of IDU males, $9.7 \%$ of non-IDU females, and 


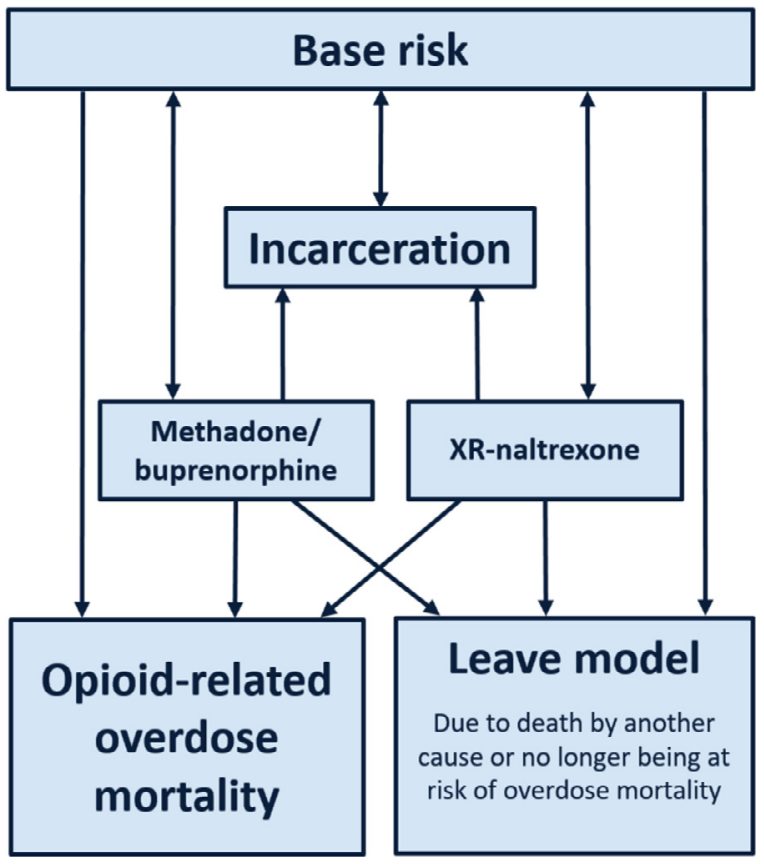

Fig. 1. Pathways for agent transition across dynamic states in the model, standard of care base case.

$1.9 \%$ of IDU females. This distribution was informed by RIDOC data and relevant literature (see appendix pp. 12-13). Recidivism risk was assigned according to agent class, with male agents and female agents having a $51 \%$ and $36 \%$ probability (respectively) of becoming re-incarcerated within 3-years following a given release from incarceration event. The distribution of sentence duration was informed by published RIDOC annual reports (appendix pp. 13). The probability of incarceration was held constant over time and did not differ by IDU or prior or current treatment status. During periods of incarceration, the probability of overdose mortality was fixed to be zero due to the low incidence of overdose mortality among persons incarcerated at the RIDOC.

Medications for opioid use disorder (MOUD)

The probability of initiating MOUD in the community was determined by agent class and remained the same across all three scenarios (Table 1). Among those initiating MOUD in the community, $99.2 \%$ were assigned to initiate methadone/buprenorphine and $0.8 \%$ were assigned to initiate XR-naltrexone; the percent initiating methadone/buprenorphine vs. XR-naltrexone was defined using publicly available, statewide, yearend 2016 treatment data (Prevent Overdose RI, 2019b). While MOUD enrollment has increased in Rhode Island in recent years (Prevent Overdose RI, 2019b), it is unclear whether these marginal increases are driven by recent structural changes (e.g., Medicaid expansion in 2014) that would not impact trends in MOUD enrollment in the long-term. Given this uncertainty, the number of agents engaged in MOUD treatment in the community was calibrated to a steady state target informed by statewide data to ensure conservative model estimates. The distributions of methadone/ buprenorphine and XR-naltrexone treatment duration were informed by the SAMHSA Treatment Episode Data Set - Discharges (TEDS-D) and prior literature (appendix pp. 14-15). Six weeks after initiating treatment, $55.0 \%$ of individuals on XR-NTX and $83.1 \%$ of individuals on methadone or buprenorphine are retained in treatment. Treatment retention then declines to $20.0 \%$ and $53.8 \%$ at one year post-treatment initiation, respectively.

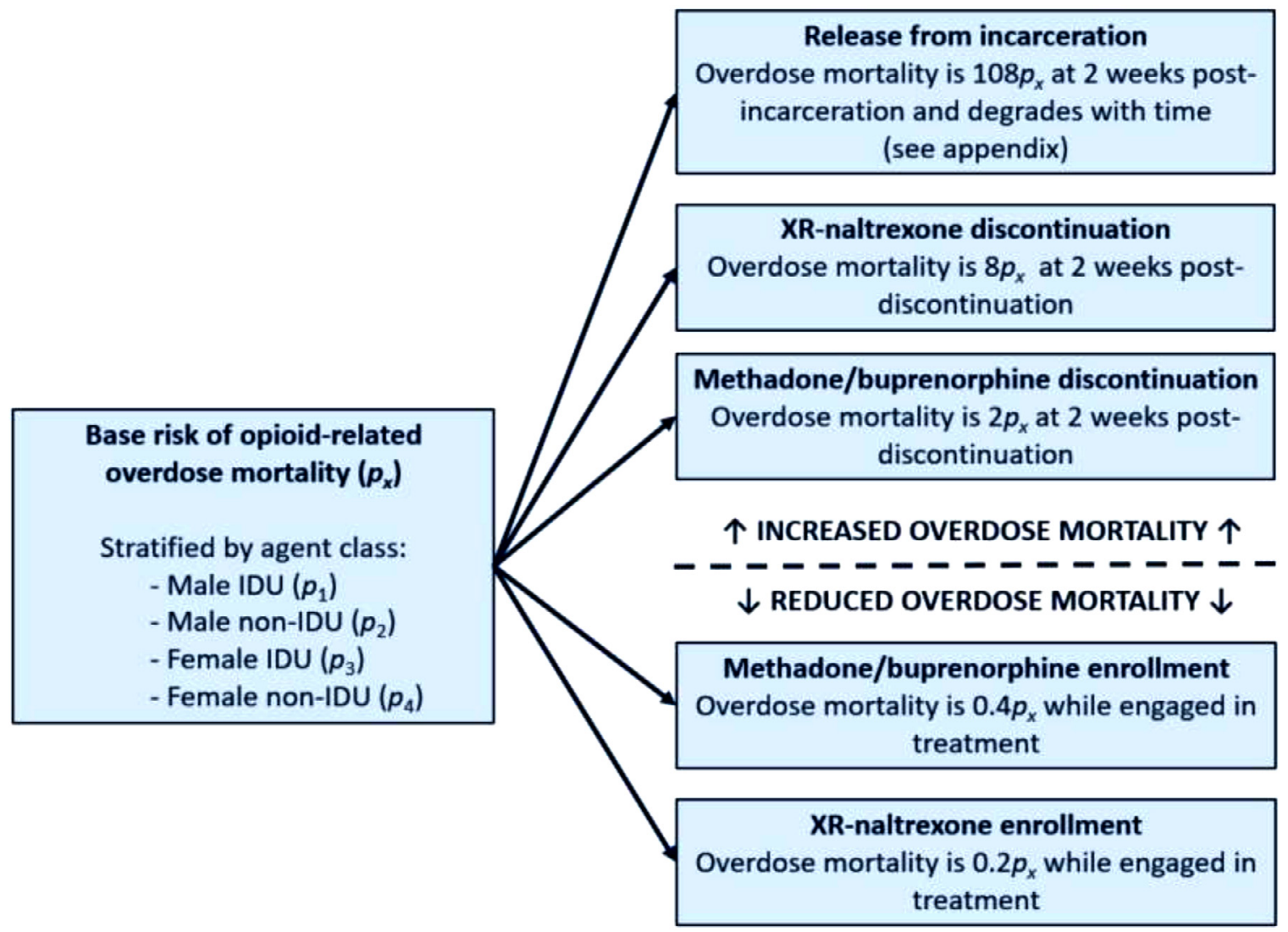

Fig. 2. Influence of dynamic agent states on base risk of opioid-related overdose mortality across agent classes $\mathrm{N}$ (ote: IDU = injection drug use; XR-naltrexone = extended-release naltrexone). 
Using de-identified RIDOC treatment enrollment data from 2016, we confirmed that less than $1 \%$ of persons who initiate MOUD are enrolled in treatment with XR-naltrexone at release; therefore, in the all three medications scenario, the current distribution of treatment allocation in the community was used (Prevent Overdose RI, 2019b), with $99.2 \%$ assigned to initiate methadone/buprenorphine and $0.8 \%$ assigned to initiate XR-naltrexone.

\section{Model scenarios}

Three scenarios were assessed in the main analyses: (1) standard of care (i.e., methadone provision to pregnant women and a 30-day taper protocol for individuals currently enrolled in methadone at time of incarceration), (2) only XR-naltrexone offered at RIDOC, and (3) all three MOUD offered at RIDOC. In the standard of care scenario, no agents were assigned to community-based MOUD in the time-step following release from incarceration. In the XR-naltrexone-only scenario, $66 \%$ of agents were assigned to community-based treatment with XRnaltrexone in the time-step following release from incarceration. This XR-naltrexone initiation rate was informed by prior research suggesting that approximately $66 \%$ of persons eligible for treatment with MOUD will opt to initiate XR-naltrexone in the absence of alternative treatment with MOUD (Di Paola et al., 2014). In the all three MOUD scenario, $100 \%$ of agents were assigned to community-based treatment with MOUD in the time-step following release from incarceration. All other parameters and processes were held constant across model scenarios. Each model scenario was simulated for 1000 iterations.

\section{Outcome measures}

The primary outcomes were the cumulative overdose mortality over 8 years (2017-2024) in the entire at-risk population and the associated percent reduction in overdose mortality relative to the standard of care. As a secondary outcome, for each scenario, we also examined the cumulative number of opioid-related overdose deaths among agents with prior-year incarceration. All outcome measures were stratified by agent class and incarceration status. Estimates are presented with $95 \%$ simulation intervals (SIs) to reflect model stochasticity (i.e., 1000 iterations for each model scenario).

\section{Calibration of main outcome measures}

In the standard of care scenario, opioid-related overdose mortality for both the entire at-risk population and for agents with prior-year incarceration was calibrated to steady state. The primary calibration targets for the model were the observed overdose mortality rates in Rhode Island in 2015. In that year, 290 opioid-related overdose deaths were observed statewide, and 51 overdose deaths occurred among persons with prior-year incarceration (Prevent Overdose RI, 2019a). In our standard of care scenario, the calibrated model produced $280 \mathrm{cu}-$ mulative overdose deaths during the first year of the simulation, and 44 of those deaths were among agents with prior-year incarceration.

In the all three MOUD scenario, calibrated model output was fairly consistent with observed overdose mortality in Rhode Island in 2017. In that year, 324 overdose deaths were observed statewide, and 10\% $(n=32)$ were observed among persons with prior-year incarceration. In our all three MOUD scenario, 28 cumulative overdose deaths occurred among agents with prior-year incarceration occurred during the first year of the simulation, accounting for $11 \%$ of all overdose deaths in the model.

This analysis did not require oversight from an institutional review board, as the model relied on publicly available, aggregate data.

\section{Sensitivity analyses}

Sensitivity analyses were conducted to assess the robustness of the primary analyses to uncertain model parameters that were likely to affect the observed impact of all three MOUD and XR-naltrexone only interventions at RIDOC; these parameters included retention in MOUD following release from incarceration, the percent of incarcerated persons who were initiated on MOUD and XR-naltrexone only following release from incarceration, overdose mortality risk during treatment with XR-naltrexone and methadone/buprenorphine, overdose risk following discontinuation of XR-naltrexone, and total population size. Parameters were considered sensitive to change if modification by $+/$ $25 \%$ from their base value produced a greater than $10 \%$ deviation in opioid-related overdose death relative to the standard of care scenario.

\section{Results}

The microsimulation model predicted that the greatest number of opioid-related overdose deaths would be observed in the standard of care scenario. In the standard of care scenario, the model predicted a cumulative number of 2385 opioid-related overdose deaths among the at-risk population in Rhode Island over the eight-year period (95\% SI: 2301-2477), and a cumulative number of 423 (95\% SI: 386-462) opioid-related overdose deaths among agents with prior-year incarceration during the same period (Table 2). The model produced relatively constant rates of opioid overdose deaths, such that the annual mortality rate in the total at-risk population was estimated to be 5.42 per 1000 person-years (95\% SI: 5.23-5.63).

Our model predicted that, relative to the standard of care scenario, reductions in opioid-related overdose mortality would be observed in both the XR-naltrexone only scenario and the all three MOUD scenario, though the greatest reductions were observed in the latter. In the entire at-risk population, the model predicted a cumulative number of 103 deaths averted over the eight-year period in the XR-naltrexone only scenario (95\% SI: $20-187$ ); this corresponds to a $4.3 \%$ population-level reduction relative to the base case over the eight-year period (95\% SI: $0.8-7.8 \%$ ). In the same population, the model predicted a cumulative number of 139 deaths averted over the eight-year period in the all three MOUD scenario (95\% SI: 53-214), corresponding to a 5.8\% reduction in the at-risk population (2.2-9.0\%).

Among agents with prior-year incarceration, the model predicted a cumulative number of 326 overdose deaths over the eight-year period in the XR-naltrexone only scenario (95\% SI: 293-360); this corresponds to approximately 12 deaths averted per year (95\% SI: $8-16)$ and a $22.8 \%$ reduction in overdose death relative to the base case $(95 \%$ SI:

Table 2.

Cumulative number of opioid-related overdose deaths, deaths averted, and percent reduction in deaths among all persons at risk in Rhode Island and among persons at risk with prior-year incarceration from 2017 to 2024 in the standard of care scenario, an extended release (XR)-naltrexone only scenario, and an all three medications for opioid use disorder (MOUD) scenario.

\begin{tabular}{|c|c|c|c|c|c|c|}
\hline \multirow[b]{2}{*}{ Scenario } & \multicolumn{3}{|l|}{ Among all persons at risk } & \multicolumn{3}{|c|}{ Among those with prior-year incarceration } \\
\hline & $\begin{array}{l}\text { Opioid-related overdose deaths, } \\
\text { mean ( } 95 \% \text { SI) }\end{array}$ & $\begin{array}{l}\text { Deaths averted } \\
(95 \% \mathrm{SI})\end{array}$ & $\begin{array}{l}\text { Percent reduction } \\
(95 \% \mathrm{SI})\end{array}$ & $\begin{array}{l}\text { Opioid-related overdose } \\
\text { deaths, mean ( } 95 \% \text { SI) }\end{array}$ & $\begin{array}{l}\text { Deaths averted } \\
(95 \% \mathrm{SI})\end{array}$ & $\begin{array}{l}\text { Percent reduction } \\
(95 \% \mathrm{SI})\end{array}$ \\
\hline Standard of care & $2385(2301-2477)$ & - & - & $423(386-462)$ & - & - \\
\hline XR-naltrexone only & $2282(2198-2365)$ & $103(20-187)$ & $4.3(0.8-7.8)$ & $326(293-360)$ & $96(63-130)$ & $22.8(14.9-30.7)$ \\
\hline All three MOUD & $2245(2171-2332)$ & $139(53-214)$ & $5.8(2.2-9.0)$ & $289(255-320)$ & $134(103-168)$ & $31.6(24.3-39.7)$ \\
\hline
\end{tabular}


14.9-30.7). In the same population, the model predicted a cumulative number of 289 overdose deaths over the eight-year period in the all three MOUD scenario (95\% SI: 255-320), corresponding to approximately 17 deaths averted per year (95\% SI: 13-21) and a $31.6 \%$ reduction in overdose deaths relative to the base case $(95 \%$ SI: 24.3-39.7\%).

\section{Sensitivity analyses}

The effects of the XR-naltrexone only intervention and the all three MOUD intervention were sensitive to the percentage of agents successfully linked to MOUD immediately following release from incarceration, with greater reductions in overdose mortality observed with higher percentages of agents linked to post-release treatment with MOUD (S8 Figure). These findings were also sensitive to post-release retention in treatment, with greater reductions in overdose mortality observed with higher treatment retention (S8 Figure). The percentage of agents successfully linked to MOUD and post-release retention in treatment were modulated concurrently across 50 analyses: 25 combinations were each explored in the XR-naltrexone only scenario and in the all three MOUD scenario. In the all three MOUD scenario, when retention in treatment was held at baseline level and the proportion of incarcerated agents released on treatment was increased to $100 \%$, we observed a $32 \%$ decrease in overdose deaths. When the proportion of incarcerated agents released on treatment was held at $0 \%$, a two-fold increase in retention in treatment produced a $10 \%$ reduction in overdose deaths. Findings were comparable in the XR-naltrexone only scenario (S8 Figure).

Overdose mortality risk during treatment with XR-naltrexone and methadone/buprenorphine (S9 Figure and S10 Figure), overdose risk following discontinuation of XR-naltrexone (S11 Table), and total population size (S12 Table) were not sensitive to change.

\section{Discussion}

This study is the first to estimate the potential population-level impact of increased access to MOUD in correctional settings on opioidrelated overdose mortality in an at-risk population. Our model predicts that providing access to all three MOUD in a correctional setting has a greater impact on overdose mortality in the community and within an at-risk population as compared to a XR-naltrexone only intervention. Relative to the standard of care, XR-naltrexone alone reduced overdose mortality by $22.8 \%$ among those with prior-year incarceration, while providing access to all three MOUD reduced overdose mortality by $31.6 \%$ among those with prior-year incarceration over the simulated eight year period.

Findings in the present study are consistent with those found in a national prospective observational study that evaluated the impact of increased access to methadone and buprenorphine in English prisons on overdose mortality post-release. Investigators in England documented a $31 \%$ reduction in drug-related poisoning deaths following release from incarceration among those who received methadone or buprenorphine while incarcerated as compared to those who did not (Marsden et al., 2017). While important differences between England and the Rhode Island setting exist, consistency between these estimates suggest that the reduction in overdose mortality projected in the current model are both realistic and attainable. Nonetheless, findings in the present study are conservative as compared to retrospective data linkage study that evaluated the impact of increased access to methadone and buprenorphine in Australian prisons on overdose mortality post-release. Investigators in New South Wales, Australia, documented a $74 \%$ reduction in accidental drug-induced deaths following release from incarceration when comparing those who received methadone or buprenorphine while incarcerated and were retained in treatment postrelease as compared to those who had not (Degenhardt et al., 2014). The greater reduction in post-release overdose deaths attained in the
Australian study underscores the importance of post-release retention in care, as those who were retained in treatment with methadone and buprenorphine were compared to those who were not. In the present study, our sensitivity analyses found that increased retention in treatment corresponded with greater reductions in overdose mortality. Improved systems to promote post-release retreatment in MOUD may yield even greater reductions in opioid-related overdose mortality postrelease than those predicted in the current model. Additionally, in the present study, we also model reductions in overdose mortality with access to XR-naltrexone, which was not evaluated in the England and Australian settings described above and may limit comparability.

Due to characteristics that are unique to Rhode Island, the present study likely underestimates the potential reduction in overdose mortality that would be observed if XR-naltrexone only or all three MOUD were implemented in correctional facilities elsewhere. First, at yearend 2016, Rhode Island had the third lowest incarceration rate in the country (Kaeble \& Cowhig, 2018). While pre-arrest diversion to treatment with MOUD is likely to produce similar reductions in overdose mortality without the harms that are associated with incarceration (Freudenberg \& Heller, 2016), those jurisdictions that incarcerate a greater proportion of persons at risk of opioid-related overdose mortality should expect to see greater reductions in overdose mortality than those projected in Rhode Island. Second, the presence of diversion programming in Rhode Island, which offers the dismissal of criminal charges to those with nonviolent felonies through participation in drug treatment and mental health programs, may produce underestimates in the potential reduction in overdose mortality that may be observed elsewhere. Third, both the XR-naltrexone only and the all three MOUD scenarios in this model were compared to the pre-2016 standard of care in Rhode Island, which allowed for the provision of methadone on a 30day taper protocol among those who were prescribed methadone prior to incarceration (Clarke et al., 2018). In the majority of prisons and jails in the US, access to MOUD is limited to women who are pregnant. Therefore, for most correctional facilities, the proportion of persons who would newly become eligible for MOUD with the implementation of either XR-naltrexone only or all three MOUD would be greater than the proportion of those who became eligible in Rhode Island. As a result, many jurisdictions could observe even greater reductions in overdose mortality relative to the previous standard of care than those projected in the current model.

Several limitations to our study warrant further investigation to ensure the validity of our model. First, the impact of an XR-naltrexone only intervention and an all three MOUD intervention varies with the level of treatment coverage and post-release retention in care achieved. While these factors were varied in the sensitivity analyses, it is important to note that the true treatment coverage and post-release treatment retention is difficult to ascertain and has important implications on the impact of both approaches. Care should be taken in transporting our model projections to other settings, as differences in MOUD availability and accessibility in the community may exist. Second, the implementation of an XR-naltrexone only intervention and an all three MOUD intervention are the only approaches explicitly modeled in this simulation; the model does not account for other harm reduction approaches or mitigating factors that might influence overdose risk behaviors or overdose mortality in the modelled population, such as naloxone distribution at release from incarceration and/or changes in the illicit drug market. Third, Rhode Island is a unique setting with a low overall level of incarceration, and the predictions made in the present model may not be representative of other locations. Fourth, heterogeneity in overdose risk in the underlying at-risk population has not been fully captured, as we were unable to identify sufficient data to further stratify overdose risk within agent classes (e.g., "high risk non-IDU male" vs "low risk non-IDU male"); however, we were able to calibrate overdose mortality across four agent classes using observed trends in Rhode Island, which is why these four strata were chosen. As such, we are confident in the overdose mortality estimates 
across these strata. Fifth, IDU status is a fixed (rather than dynamic) agent state in the current model because we were unable to identify sufficient data to reliably reproduce an accurate rate of transitions (e.g., from non-IDU to IDU) in Rhode Island. At the individual level, some modulation between IDU states would be expected; however, implementing these individualized transitions in the absence of data would introduce error in model estimates at the population-level and reduce model validity. Sixth, the distribution of sentence lengths that were implemented in this model were calibrated to all persons incarcerated in Rhode Island, of whom, approximately a third are estimated to be at risk of overdose. This is a limitation of the model, as the distribution of sentence duration for this population has not yet been assessed beyond 6 months (Rich et al., 2015); nonetheless, we believe that these estimates are reasonably representative of those that would be observed in the subset of individuals who are at risk of overdose were these measured and calculated in the real world. Seventh, there is some evidence of an increased risk of opioid-related overdose mortality during the initial two-week induction phase onto treatment with methadone that is not explicitly reflected by model processes (Sordo et al., 2017). Nonetheless, we believe that this period of increased risk is captured in our parameterization of the overall risk of opioid-related overdose mortality during treatment with methadone/buprenorphine, which was also informed by systematic reviews (Degenhardt et al., 2011; Mathers et al., 2013). Finally, while local data were used to parameterize the model whenever possible, several input parameters were derived from multiple sources, which could introduce bias and adversely affect the accuracy and generalizability of the model outputs.

Providing access to all three MOUD in prisons and jails has the potential to avert more opioid-related overdose death than the pre2016 standard of care in Rhode Island or providing access to XR-naltrexone alone, which has poorer long-term treatment adherence and higher mortality rates after treatment discontinuation. The predicted impact of either approach varies with treatment coverage and post-release treatment retention. Therefore, jurisdictions that have limited MOUD treatment capacity in the community should expand both corrections-based and community-based MOUD treatment concurrently.

\section{Contributors}

AM, JWA, SEB, and BDLM conceptualized the study and design. AM developed initial drafts of the manuscript. AM and JWA developed initial drafts of the supplemental files. SEB implemented the model. AM conducted all analyses. JWA, SEB, LBR, RAM, JGC, TCG, JDR, and BDLM provided iterative and substantive feedback on the overall approach and all drafts of the manuscript. All authors have approved the final manuscript.

\section{CRediT authorship contribution statement}

Alexandria Macmadu: Conceptualization, Methodology, Software, Validation, Formal analysis, Writing - original draft, Visualization. Joëlla W. Adams: Conceptualization, Methodology, Writing - original draft, Supervision. S.E. Bessey: Conceptualization, Methodology, Software, Validation, Writing - review \& editing, Project administration. Lauren Brinkley-Rubinstein: Writing - review \& editing. Rosemarie A. Martin: Data curation, Writing - review \& editing. Jennifer G. Clarke: Writing - review \& editing. Traci C. Green: Writing - review \& editing. Josiah D. Rich: Conceptualization, Writing - review \& editing, Supervision. Brandon D.L. Marshall: Conceptualization, Methodology, Resources, Writing - review \& editing, Supervision.

\section{Declaration of Competing Interest}

No conflicts declared.

\section{Acknowledgements}

The work of AM, JR, and BDLM was supported by grants from the National Institute on Drug Abuse (K24DA022112 and R21DA044443). Their work was also supported by the COBRE on Opioids and Overdose, which is funded by the National Institute of General Medical Sciences of the National Institutes of Health (P20GM125507). The content is solely the responsibility of the authors and does not necessarily represent the official views of the National Institutes of Health.

\section{Supplementary materials}

Supplementary material associated with this article can be found, in the online version, at doi:10.1016/j.drugpo.2020.102841.

\section{References}

Arnold, K. F., Harrison, W. J., Heppenstall, A. J., \& Gilthorpe, M. S. (2019). DAG-informed regression modelling, agent-based modelling and microsimulation modelling: A critical comparison of methods for causal inference. International Journal of Epidemiology, 48(1), 243-253.

Binswanger, I. A., Blatchford, P. J., Mueller, S. R., \& Stern, M. F. (2013). Mortality after prison release: Opioid overdose and other causes of death, risk factors, and time trends from 1999 to 2009. Annals of Internal Medicine, 159(9), 592-600.

Binswanger, I. A., Stern, M. F., Deyo, R. A., Heagerty, P. J., Cheadle, A., Elmore, J. G., et al. (2007). Release from prison-A high risk of death for former inmates. New England Journal of Medicine, 356(2), 157-165.

Clarke, J. G., Martin, R. A., Gresko, S. A., \& Rich, J. D. (2018). The first comprehensive program for opioid use disorder in a us statewide correctional system. American Public Health Association.

Degenhardt, L., Bucello, C., Mathers, B., Briegleb, C., Ali, H., Hickman, M., et al. (2011). Mortality among regular or dependent users of heroin and other opioids: A systematic review and meta-analysis of cohort studies. Addiction (Abingdon, England), 106(1), $32-51$.

Degenhardt, L., Larney, S., Kimber, J., Gisev, N., Farrell, M., Dobbins, T., et al. (2014). The impact of opioid substitution therapy on mortality post-release from prison: Retrospective data linkage study. Addiction (Abingdon, England), 109(8), 1306-1317.

Di Paola, A., Lincoln, T., Skiest, D. J., Desabrais, M., Altice, F. L., \& Springer, S. A. (2014). Design and methods of a double blind randomized placebo-controlled trial of extended-release naltrexone for HIV-infected, opioid dependent prisoners and jail detainees who are transitioning to the community. Contemporary Clinical Trials, 39(2), $256-268$.

Digiusto, E., Shakeshaft, A., Ritter, A., O'Brien, S., Mattick, R. P., \& Group, N. R. (2004). Serious adverse events in the Australian National Evaluation of Pharmacotherapies for Opioid Dependence (NEPOD). Addiction (Abingdon, England), 99(4), 450-460.

Fingerhood, M. I., King, V. L., Brooner, R. K., \& Rastegar, D. A. (2014). A comparison of characteristics and outcomes of opioid-dependent patients initiating office-based buprenorphine or methadone maintenance treatment. Substance Abuse, 35(2), 122-126.

Freudenberg, N., \& Heller, D. (2016). A review of opportunities to improve the health of people involved in the criminal justice system in the United States. Annual Review of Public health, 37, 313-333.

Gibson, A. E., \& Degenhardt, L. J. (2007). Mortality related to pharmacotherapies for opioid dependence: A comparative analysis of coronial records. Drug and Alcohol Review, 26(4), 405-410.

Havens, J. R., Oser, C. B., Knudsen, H. K., Lofwall, M., Stoops, W. W., \& Walsh, S. L. (2011). Individual and network factors associated with non-fatal overdose among rural Appalachian drug users. Drug and Alcohol Dependence, 115(1-2), 107-112.

Hedrich, D., Alves, P., Farrell, M., Stöver, H., Møller, L., \& Mayet, S. (2012). The effectiveness of opioid maintenance treatment in prison settings: A systematic review. Addiction (Abingdon, England), 107(3), 501-517.

Heimer, R., Catania, H., Newman, R. G., Zambrano, J., Brunet, A., \& Ortiz, A. M. (2006) Methadone maintenance in prison: Evaluation of a pilot program in Puerto Rico. Drug and Alcohol Dependence, 83(2), 122-129.

Joudrey, P. J., Khan, M. R., Wang, E. A., Scheidell, J. D., Edelman, E. J., McInnes, D. K., et al. (2019). A conceptual model for understanding post-release opioid-related overdose risk. Addiction Science \& Clinical Practice, 14(1), 1-14.

Kaeble, D., \& Cowhig, M. (2018). Correctional populations in the united states, 2016. (NCJ 251211). Washington, DC: US Department of Justice, Office of Justice Programs, Bureau of Justice Statistics.

Kinlock, T. W., Gordon, M. S., Schwartz, R. P., Fitzgerald, T. T., \& O'Grady, K. E. (2009). A randomized clinical trial of methadone maintenance for prisoners: Results at 12 months postrelease. Journal of Substance Abuse Treatment, 37(3), 277-285.

Kinner, S. A., Milloy, M., Wood, E., Qi, J., Zhang, R., \& Kerr, T. (2012). Incidence and risk factors for non-fatal overdose among a cohort of recently incarcerated illicit drug users. Addictive Behaviors, 37(6), 691-696.

Krupitsky, E., Nunes, E. V., Ling, W., Illeperuma, A., Gastfriend, D. R., \& Silverman, B. L. (2011). Injectable extended-release naltrexone for opioid dependence: A doubleblind, placebo-controlled, multicentre randomised trial. The Lancet, 377(9776), 1506-1513. 
Lam, J. A., Lee, H. I. S., Truong, A. Q., Macmadu, A., Clarke, J. G., Rich, J., et al. (2019). Brief video intervention to improve attitudes throughout medications for opioid use disorder in a correctional setting. Journal of Substance Abuse Treatment, 104, 28-33.

Larochelle, M. R., Bernson, D., Land, T., Stopka, T. J., Wang, N., Xuan, Z., et al. (2018). Medication for opioid use disorder after nonfatal opioid overdose and association with mortality: A cohort study. Annals of Internal Medicine, 169(3), 137-145.

Lee, J. D., Friedmann, P. D., Kinlock, T. W., Nunes, E. V., Boney, T. Y., Hoskinson, R. A., $\mathrm{Jr}$, et al. (2016). Extended-release naltrexone to prevent opioid relapse in criminal justice offenders. New England Journal of Medicine, 374(13), 1232-1242.

Lee, J. D., Nunes, E. V., Jr, Novo, P., Bachrach, K., Bailey, G. L., Bhatt, S., et al. (2018). Comparative effectiveness of extended-release naltrexone versus buprenorphine-naloxone for opioid relapse prevention (X: BOT): A multicentre, open-label, randomised controlled trial. The Lancet, 391(10118), 309-318.

Marsden, J., Stillwell, G., Jones, H., Cooper, A., Eastwood, B., Farrell, M., et al. (2017) Does exposure to opioid substitution treatment in prison reduce the risk of death after release? A national prospective observational study in England. Addiction (Abingdon, England), 112(8), 1408-1418.

Mathers, B. M., Degenhardt, L., Bucello, C., Lemon, J., Wiessing, L., \& Hickman, M. (2013). Mortality among people who inject drugs: A systematic review and metaanalysis. Bulletin of the World Health Organization, 91, 102-123.

Merrall, E. L., Kariminia, A., Binswanger, I. A., Hobbs, M. S., Farrell, M., Marsden, J., et al. (2010). Meta-analysis of drug-related deaths soon after release from prison. Addiction (Abingdon, England), 105(9), 1545-1554.

Møller, L., Matic, S., van Den Bergh, B., Moloney, K., Hayton, P., \& Gatherer, A. (2010). Acute drug-related mortality of people recently released from prisons. Public Health, 124(11), 637-639.

Moore, K. E., Roberts, W., Reid, H. H., Smith, K. M., Oberleitner, L. M., \& McKee, S. A. (2019). Effectiveness of medication assisted treatment for opioid use in prison and jail settings: A meta-analysis and systematic review. Journal of Substance Abuse Treatment, 99, 32-43.

National Academies of Sciences, E., \& Medicine. (2019). Medications for opioid use disorder save lives. National Academies Press.

Prevent Overdose, R.I. (2019). Former inmates are at high risk of overdose. Retrieved from https://preventoverdoseri.org/overdose-deaths/.
Prevent Overdose, R.I. (.2019). Medication-Assisted Treatment Data. Available at: https://preventoverdoseri.org/medication-assisted-therapy/.

Prevent Overdose, R.I. (.2019). Overdose Death Data. Available at: https:// preventoverdoseri.org/overdose-deaths/.

Rhode Island Department of Corrections. (2017). Monthly Offender Statistics and Reports. Available at: http://www.doc.ri.gov/administration/planning/statistics_reports.php.

Rhode Island Department of Health. (2019). Drug Overdose Deaths. Available at: https:// health.ri.gov/data/drugoverdoses/.

Rich, J. D., McKenzie, M., Larney, S., Wong, J. B., Tran, L., Clarke, J., et al. (2015). Methadone continuation versus forced withdrawal on incarceration in a combined US prison and jail: A randomised, open-label trial. The Lancet, 386(9991), 350-359.

Saucier, R., Wolfe, D., \& Dasgupta, N. (2018). Review of case narratives from fatal overdoses associated with injectable naltrexone for opioid dependence. Drug Safety, 41(10), 981-988.

Scholl, L., Seth, P., Kariisa, M., Wilson, N., \& Baldwin, G. (2019). Drug and opioid-involved overdose deaths-United States, 2013-2017. Morbidity and Mortality Weekly Report, 67(5152), 1419.

Substance Abuse and Mental Health Data Archive. (2015-2016). 2-Year Restricted-use Data Analysis System.

Sordo, L., Barrio, G., Bravo, M. J., Indave, B. I., Degenhardt, L., Wiessing, L., ... PastorBarriuso, R. (2017). Mortality risk during and after opioid substitution treatment: Systematic review and meta-analysis of cohort studies. BMJ (Clinical research ed.), 357, J1550.

Vestal, C. (2018). New momentum for addiction treatment behind bars. The Pew Chartiable Trusts.. https://www.pewtrusts.org/en/research-and-analysis/blogs/ stateline/2018/04/04/new-momentum-for-addiction-treatment-behind-bars Accessed April 25, 2020.

Wilson, N., Kariisa, M., Seth, P., Smith, H., \& Davis, N. (2020). Drug and opioid-involved overdose deaths-United States, 2017-2018. MMWR. Morbidity and Mortality Weekly Report, 69.

Zaller, N., McKenzie, M., Friedmann, P. D., Green, T. C., McGowan, S., \& Rich, J. D. (2013). Initiation of buprenorphine during incarceration and retention in treatment upon release. Journal of Substance Abuse Treatment, 45(2), 222-226. 